\title{
Severe rhabdomyolysis reveals peripheral hypothyroidism
}

\section{Siham Belmourida, Hind Palamino, Mariame Meziane, Nadia Ismaili, Laila Benzekri, Baderdine Hassam, Karima Senouci}

Departement of Dermatology-Venerology, Mohammed V University, Ibn Sina Hospital, Rabat, Morocco

Corresponding author: Dr. Siham Belmourida, E-mail: belmouridasiham2017@gmail.com

Sir,

Rhabdomyolysis on hypothyroidism is a rare phenomenon to our knowledge, only a few cases are described in the literature [1].

We report a case of severe rhabdomyolysis secondary to a deep hypothyroidism.

A 60-year-old woman with no notable pathology, had been suffering from myalgia, asthenia, anorexia and constipation for three years. She had consulted in dermatology for bilateral palpebral edema with diffuse muscular cramps.

The clinical examination revealed an asthenic patient, bradycardia at $60 \mathrm{~b} / \mathrm{min}$, generalized myxedema (Figs. 1 and 2) with myalgia and psychomotor retardation. Examination of the thyroid lodge did not reveal goiter.

A first assessment showed a major increase in muscle lysis enzymes (creatine phosphokinase $(\mathrm{CPK})$ at 30 times normal, aldolases and transaminases). The diagnosis of hypothyroid myopathy was suspected in the presence of clinical signs of hypothyroidism and confirmed by hormonal assays. The cardiac ultrasound revealed an effusion of low abundance. The antiperoxidase antibodies were very high and the diagnosis of Hashimoto's thyroiditis was retained.

The hormonal replacement therapy allowed the normalization of thyroid hormones and the regression of muscle signs.

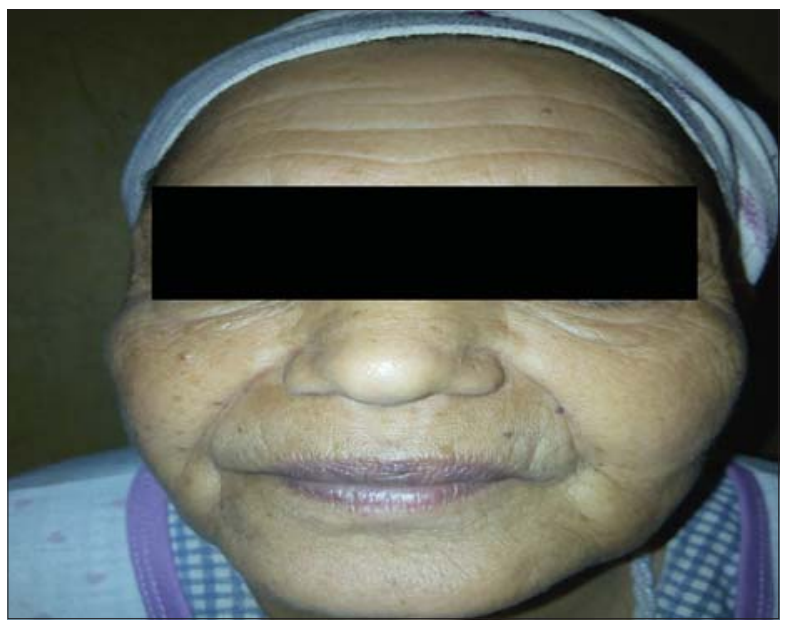

Figure 1 : Infiltration of the face secondary to deep hypothyroidosis.

Rhabdomyolysis secondary to hypothyroidism is a rare phenomenon. It is an acute myonecrosis manifested by a serum elevation of the enzymes of muscle lysis $[1,2]$.

However, cases of major elevation of CPK with or without renal insufficiency during hypothyroid myopathy are exceptional, as is the case of our patient. The origin of this increase is poorly known, it could be an increase in muscle catabolism associated with a decrease in the clearance of enzymes. The degradation of renal function is possible and it's secondary to rhabdomyolysis. However, before retaining the diagnosis of hypothyroid myopathy before a table of rhabdomyolysis associated with acute renal failure, other classical etiologies must be eliminated [3].

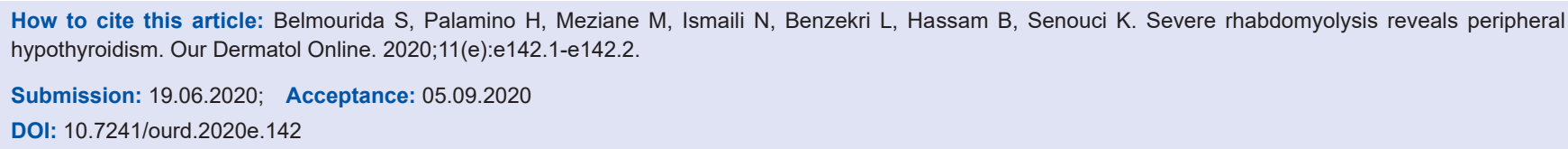




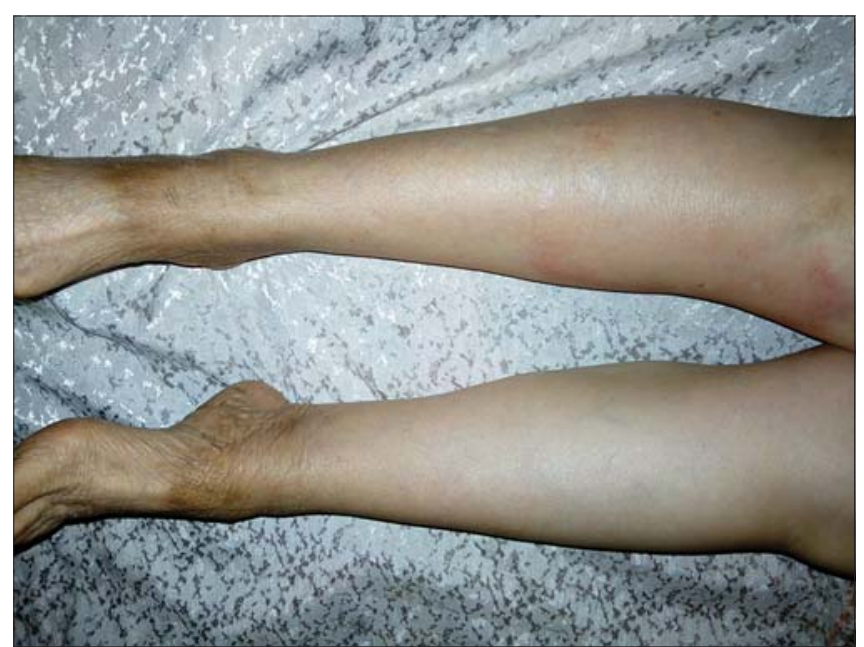

Figure 2 : Lower limb edema with significant skin dryness .

Hormonal substitution is imperative, hemodialysis may be necessary in the case of severe hyperkalemia or in cases of oligo-anuria or anuria [1-3].

In front of myalgia with muscle weakness accompanied by a high rate of muscle enzymes, think of hypothyroidism.

\section{Consent}

The examination of the patient was conducted according to the principles of the Declaration of Helsinki.

The authors certify that they have obtained all appropriate patient consent forms, in which the patients have given consent for images and other clinical information to be included in the journal. The patients understand that their names and initials will not be published and due effort will be made to conceal their identity, but that anonymity cannot be guaranteed.

\section{REFERENCES}

1. Djermane A. Hypothyroïdie et rhabdomyolyse : à propos d'un cas. Ann Endocrinol. 2014.07.747.

2. Nooshin S, Endre A, Iqbal M, and Gary JT. Rhabdomyolysis in a Patient with Severe Hypothyroidism. Am J Case Rep. 2017;18:912-8.

3. Sindoni A, Rodolico C, Pappalardo MA, Portaro S, Benvenga S. Hypothyroid myopathy: A peculiar clinical presentation of thyroid failure. Review of the literature. Rev Endocr Metab Disord. 2016;17:499-519.

Copyright by Siham Belmourida, et al. This is an open-access article distributed under the terms of the Creative Commons Attribution License, which permits unrestricted use, distribution, and reproduction in any medium, provided the original author and source are credited.

Source of Support: Nil, Conflict of Interest: None declared. 